\title{
Oral albendazole in the management of extraocular cysticercosis
}

\author{
Ramanjit Sihota, Santosh G Honavar
}

\begin{abstract}
Surgical removal of extraocular cysticerci is fraught with complications. The effect of oral albendazole in such cases has been evaluated in a randomised, controlled, clinical trial. Of 24 ultrasonographically diagnosed, and ELISA positive cases of extraocular cysticerci, 12 received oral albendazole $15 \mathrm{mg} / \mathrm{kg}$ once daily for 1 month, while the 12 controls received a placebo. Marked clinical improvement was seen in all the cases in the treatment group at 4 weeks, with collapse of the cyst at 6 weeks $(75 \%)$, and complete disappearance at 3 months $(100 \%)$. No clinical or ultrasonographical change was noted in the control group. A trial of medical management with oral albendazole merits consideration in cases of extraocular cysticerci.
\end{abstract}

(Brf Ophthalmol 1994; 78: 621-623)

Cysticercosis commonly affects the eye and extraocular structures. Intraocular cysticerci are diagnosed more readily because of their visibility whereas the diagnosis of extraocular cysticerci was largely speculative, until the advent of advanced imaging modalities like ultrasonography and computed tomography (CT), and the enzyme linked immunosorbent assay (ELISA) technique. The increased availability of ultrasonography enables us to detect many lesions caused by cysticerci in the orbit, which were higherto labelled as forms of orbital pseudotumours. The use of systemic corticosteroids and anti-inflammatory drugs in such cases temporarily relieves symptoms by suppressing pericystic inflammation. Surgery to remove these cysts is fraught with risks owing to adhesion to adjacent vital structures and frequent rupture. ${ }^{1}$

Neurocysticercosis has been treated successfully with albendazole, an imidazole, the [5-(propylthio)-1H-benzimidazole-2yl] carbamic acid methyl ester and with praziquantel..$^{2-6}$ Sotelo et al have compared the two drugs and found albendazole to be more effective than praziquantel and also less expensive. ${ }^{23}$

We have studied the efficacy of oral albendazole in the management of extraocular cysticercosis in this randomised controlled clinical trial.

\section{Patients and methods}

Twenty four consecutive patients diagnosed by ultrasonography to have a possible cysticercus cyst and confirmed by a positive ELISA test in the serum were enrolled for this study. Baseline examination produced details of clinical history including diet history; record of Snellen visual acuity; anterior segment examination; indirect ophthalmoscopy; detailed orthoptic examination including diplopia charting, Hess screen; Hertel's exophthalmometry; orbital B scan ultrasonography; immersion B scan ultrasonography for subconjunctival cysts (Ophthascan-S, Biophysic); skull $x$ ray; CT of skull; serum ELISA for cysticercosis; and clinical photographs. Informed consent to participate in the study was given by each patient. If the patients were previously treated with corticosteroids or antiinflammatory drugs, they were observed off all therapy for a period of at least 2 months before being included in the study. Patients with each type of clinical presentation such as proptosis, ocular motility disorders, and subconjunctival cyst were randomly assigned to receive either oral albendazole in the dose of $15 \mathrm{mg} / \mathrm{kg}$ body weight once a day, or multivitamin capsules as a placebo, for a period of 1 month. All the patients were followed up at intervals of 2 weeks for 2 months and then monthly till the end of the sixth month, and at each visit, clinical examination, exophthalmometry, diplopia charting, Hess charting, and ultrasonography were repeated. All the patients complied with the follow up schedule.

\section{Observations}

Patient characteristics are shown in Table 1. The commonest mode of presentation was proptosis (12 patients, $50 \%$ ), followed by ocular motility disorders (10 patients, 42\%), and subconjunctival cyst (two patients, 8\%). All the patients with ocular motility disorders complained of diplopia. Twelve patients had previously received a course of systemic corticosteroids for durations varying from 1 to 3 months, with temporary relief in symptoms.

The presence of a well defined cyst, having clear contents and visible scolex, was documented on ultrasound in all the patients (Fig 1). The size of the cysts ranged from 5.4 to $13.3 \mathrm{~mm}$ (mean $7 \cdot 5$ (SD 2.23) $\mathrm{mm}$ ). All the patients had cysts within or attached to the extraocular muscles. The commonest muscle involved was the medial rectus (16 patients, 67\%). Associated

Table 1 Patient characteristics

\begin{tabular}{lll}
\hline & $\begin{array}{l}\text { Albendazole } \\
\text { group } \\
(n=12)\end{array}$ & $\begin{array}{l}\text { Placebo } \\
\text { group } \\
(n=12)\end{array}$ \\
\hline Age (years): & $18(4 \cdot 79)$ & $20(5 \cdot 29)$ \\
$\quad$ Mean (SD) & $6-22$ & $8-25$ \\
Range & $5: 7$ & $6: 6$ \\
Male:female & $6(50)$ & $6(50)$ \\
Presentation (n (\%)): & $5(42)$ & $5(42)$ \\
Proptosis & $1(8)$ & $1(8)$ \\
Motility disorder & $7 \cdot 65(2 \cdot 39)$ & $7 \cdot 35(2 \cdot 15)$ \\
Subconjunctival cyst & $5 \cdot 4-13 \cdot 3$ & $5 \cdot 4-12 \cdot 9$ \\
Cyst size (mm): & & \\
$\quad$ Mean (SD) & & \\
Range & &
\end{tabular} \\ Centre for Ophthalmic \\ Sciences, All India \\ Institute of Medical \\ India \\ S G Honava \\ April 1994
}




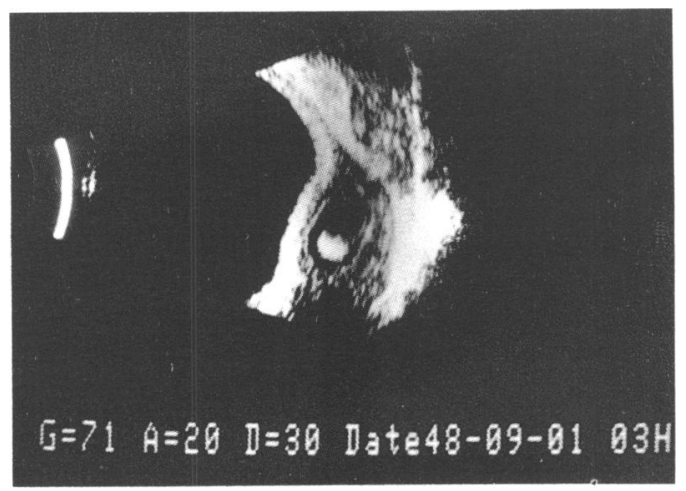

Figure 1 Cysticercus cyst in the inferior rectus muscle with well defined wall and scolex.

neurocysticercosis was detected in four patients $(16 \cdot 7 \%)$.

After 2 weeks of albendazole therapy, all the patients reported a significant improvement in their symptoms, be they diplopia, proptosis, or a subconjunctival mass. Ultrasonography showed obscuration of the cyst wall in areas. The scolex was visible, though with decreased clarity and the clear space within the cyst was full of random echoes (Fig 2).

At the fourth week, symptomatic improvement was reported by all patients, and supported by repeat diplopia charting, Hess charting. Ultrasonography showed an obliteration of the capsule of the cyst and echoes suggestive of marked infiltration of the adjacent extraocular muscle and orbital fat. The scolex could no longer be pinpointed accurately in nine of the 12 patients owing to the presence of dense, diffuse, intracystic echoes.

Six weeks after the initiation of albendazole therapy, a collapsed cyst was seen in nine of the 12 patients, while in the rest a fusiform dilatation of the adjacent muscle was the only evidence of the parasite. Further improvements in clinical parameters were noted. However, in patients complaining of diplopia, this persisted in extremes of gaze with an attendant positive forced duction test. After 8 weeks, no ultrasonographic evidence of the cyst was seen in any of the patients but diffuse echoes suggestive of infiltration of the tissues persisted. Diplopia still persisted in extremes of gaze. By 3 months only mild infiltration of the muscles suggested by minimal diffuse echoes remained as a legacy of the cysticercus. No further changes in the ultrasound or clinical parameters were noted on further follow up.

We measured ultrasonographically the size of the cyst initially, but were unable to continue this as the cyst collapsed and was indistinguishable from the surrounding diffuse echoes after 4 weeks.

In the albendazole treatment group, one patient had severe pain and oedema of lids 2 days after the treatment was started. Four others complained of pain on ocular movement for 2-3 days in the first week, without any associated inflammatory signs being visible. One patient again complained of mild pain at the 6 week visit. These symptoms subsided completely on treatment with oral ibuprofen $200-400 \mathrm{mg}$ thrice a day for 2-5 days. No systemic side effects of albendazole were encountered.

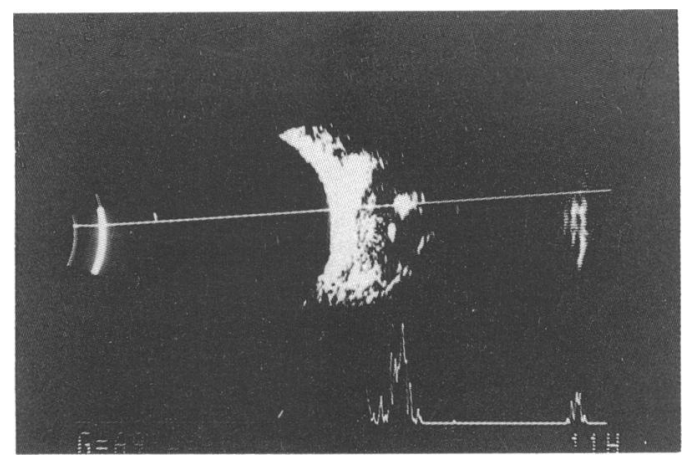

Figure 2 After 2 weeks of albendazole therapy, the cyst walls and scolex are ill defined. There are intracystic random echoes and evidence of surrounding inflammation in the inferior rectus muscle.

No changes in the initial symptoms, clinical picture, baseline parameters, or the ultrasound picture were seen in the control group.

Three of the four patients with neurocysticercosis showed a resolution of the cyst on repeat CT of the skull at 3 months.

\section{Discussion}

Taenia solium has a worldwide prevalence but is endemic in Mexico, Africa, East Europe, south east Asia, and Central and South America. ${ }^{7}$ Ocular or adnexal involvement occurs in 13-46\% of infected patients ${ }^{89}$ - the most common site of localisation being the posterior segment of the eye in most Western reports and the ocular adnexa in most Indian ones. ${ }^{710}{ }^{11}$ On review of all the documented cases of ocular and adnexal cysticercosis, Kruger-Leite et al ${ }^{12}$ found that $35 \%$ of the cysts were reported in the subretinal space, $22 \%$ in the vitreous, $22 \%$ in the subconjunctival space, $5 \%$ in the anterior segment, and only $1 \%$ in the orbit.

The advent of newer high resolution ultrasonography machines has enabled us to diagnose accurately cases that were earlier labelled as orbital pseudotumours or acquired oculomotor anomalies. The first few cases seen were considered an unusual presentation of ophthalmic cysticercosis and we even surgically excised two lesions for histopathological confirmation when there was no significant change in the clinical or ultrasonographic picture after a 3 month course of systemic corticosteroids. Subsequently we began to recognise more and more of these lesions, as also the fact that corticosteroids caused, at best, a temporary remission in the symptoms. Although some workers have reported gratifying results with surgical excision of orbital cysticerci, ${ }^{13}$ it is a general impression that these cysts are serious surgical challenges because of their size, attachment to important orbital structures, situation in the depth of the orbit, and propensity to rupture intraoperatively.' Surgery is also fraught with inevitable postoperative fibrotic response, which may result in restrictive ocular motility disorders. It is believed that anthelmintic drugs have little effect on orbital cysticerci owing to poor drug penetration. ' Since all the lesions we found were associated with extraocular muscles implying reasonable vascularity, we decided to evaluate the efficacy of anthelmintic drugs in such cases.

Praziquantel has been reported to exert a toxic 
but reversible effect on intravitreal cysticercosis. ${ }^{14} \mathrm{~A}$ study of the literature regarding the medical therapy of neurocysticercosis suggested that albendazole was very useful and more effective than praziquantel..$^{23}$

We have evaluated the efficacy of oral albendazole in the management of extraocular cysticercosis in a randomised controlled clinical trial. We followed the therapeutic regimen successfully adopted by Sotelo et al, ${ }^{2}$ using $15 \mathrm{mg} / \mathrm{kg}$ body weight of albendazole once a day for 1 month. Our results were extremely gratifying with a complete disappearance of all cysts, a total resolution of proptosis and subconjunctival cysts, and minimal residual oculomotor restriction at the end of the study. In contrast, all the controls showed no change in the size or characteristics of the cysts over the period of observation. The response of neurocysticercosis to albendazole has been reported in the range of 70$80 \%$. The better vascularity of extraocular muscles and orbital tissues, and therefore greater bioavailability of the drug may be responsible for the excellent results in our study.

Sotelo et al report that the natural history of cysticercosis inevitably results in granuloma formation. ${ }^{2}$ On a follow up of medically treated neurocysticercosis, CT showed an absence of granulomas in $80 \%$ of the patients. ${ }^{23}$ In another study, Sotelo et al found that lesions surrounded by contrast enhancement of the CT image spontaneously improved over a long period of time, but not those without enhancement. ${ }^{15}$ Kramer et $a l$, on the other hand, feel that even nonenhancing, non-oedematous lesions on CT, disappear with time in about $55 \%$ of those involved. ${ }^{16}$

The orbital ultrasound gives us a more precise picture of the cyst and its surroundings. Our study only included cases with well defined cysts, with clear contents, and a sharply outlined scolex. Controls showed no significant change with time, while albendazole treated patients all showed evidence of progressively increasing inflammation both around and within the cyst up to the fourth week. This was accompanied by a disintegration of the structure of the cyst, a fuzzy thickening of and collapse of the walls, increased intracystic echoes, and a disappearance of the scolex. Over the next 2 months, the inflammation gradually subsided, leaving minimal infiltration of the extraocular muscle involved. Zenteno et al have described a similar regression pattern in cerebral cysticerci seen on magnetic resonance imaging. ${ }^{17}$
Shorter courses of albendazole of 3 days and 8 days have also been shown to be effective in neurocysticercosis, ${ }^{18} 19$ and are presently being investigated by us. The utility of a combination of albendazole with corticosteroids or nonsteroidal anti-inflammatory drugs to suppress associated inflammatory response during the period of therapy needs to be investigated.

It would be wise to investigate all cases of subacute proptosis, cases of presumed pseudotumour not adequately responding to systemic corticosteroids, acquired oculomotor disorders, and subconjunctival cysts with ultrasonography in areas in which cysticercosis is endemic. On detection by ultrasonography and confirmation by serum ELISA for cysticercosis, a trial of oral albendazole therapy merits consideration before any surgical intervention.

1 Shields JA. Diagnosis and management of orbital tumors. Philadelphia: W B Saunders, 1989: 117-20.

2 Sotelo J, Escobedo F, Penagos P. Albendazole vs praziquantel for therapy for neurocysticercosis - a controlled trial. Arch Neurol 1988; 45: 532-4.

3 Sotelo J, Del Brutto OH, Penagos P. Comparison of therapeutic regimen of anticysticercal drugs for parenchymal brain cysticercosis. $\mathcal{F}$ Neurol $1990 ; 237$ : 69-72.

4 del Brutto OH, Sotelo J. Albendazole therapy for subarachnoid and ventricular cysticercosis. $\mathcal{F}$ Neurosurg 1990; 72: 816-7.

5 Agapejev S, Meira DA, Barraviera B, Machado JM, Marques Pereira PC, Mendes RP, et al. Neurocysticercosis: treatment with albendazole and dextrochlorpheniramine. Trans $R$ Soc Trop Med Hyg 1989; 83: 377-83.

6 Moodley M, Moosa A. Treatment of neurocysticercosis: is ? praziquantel the new hope? Lancet 1989; i: 262-3.

7 Cano MR. Ocular cysticercosis. In: Ryan SJ, ed. Retina. Vol 2. St Louis: Mosby, 1989: 583-7.

8 Duke-Elder S, Perkins ES. System of ophthalmology. Vol 9. Diseases of the uveal tract. St Louis: Mosby, 1966.

9 Mais FA. Criocirugia na cisticercose ocular. Rev Bras Ophthalmol 1969; 28: 99-106.

10 Malik SRK, Gupta AK, Choudhry S. Ocular cysticercosis. Am F Ophthalmol 1968; 66: 1168 .

11 Sivareddy P, Satyendran OM. Ocular cysticercosis. Am F Ophthalmol 1964; 57: 664.

12 Kruger-Leite E, Jalkh AE, Quiroz H, Schepens CL. Intraruger-Leite E, Jalkh AE, Quiroz H, Schepens CL. Int
ocular cysticercosis. Am f Ophthalmol 1985; 99: 252-7.

13 Sen DK. Cysticercus cellulosae in the lacrimal gland, orbit and eyelid. Acta Ophthalmol 1980; 58: 144-7.

14 Kestelyn P, Taelman H. Effect of prazquantel on intraocular cysticercosis: a case report. $\mathrm{Br} \mathcal{F}$ Ophthalmol 1985; 69: 788-90.

15 Sotelo J, Guerrevo J, Rubio R. Neurocysticercosis: a new classification based on active and inactive forms - a study of 753 cases. Arch Intern Med 1985; 145: 442-5.

16 Kramer LD, Locks GE, Byrd SF, Darybagi J. Cerebral cysticercosis - documentation of natural history by CT. Radiology 1989; 171: 459-62.

17 Zenteno MA, Del Brutto OH, Honang B, Sotelo J, Penagos P. Neurocysticercosis an pays d'endemic (Mexique, interet de l'IRM). Presented at the annual meeting of the Societe Francaise de Neuro-radiologie, Paris, France, 1987.

18 Sotelo J, Penagos P, Escobedo F, Del Brutto OH. Short course of albendazole therapy for neurocysticercosis. Arch Neurol 1988; 45: 1130-3.

19 Alarcon F, Escalente L, Duenas G, Montalvo M, Roman M. neurocysticercosis - short course of treatment with albendazole. Arch Neurol 1989; 46: 1231-6. 